\title{
Atmospheric effect corrections of MuSTAnG data
}

\author{
Mary Zazyan ${ }^{1,2, *}$, Marina Ganeva ${ }^{1,3}$, Marina Berkova ${ }^{4}$, Victor Yanke ${ }^{4}$, and Rainer Hippler ${ }^{1}$ \\ 1 Institute of Physics, Ernst-Moritz-Arndt University of Greifswald, Felix-Hausdorff-Str. 6, 17487 Greifswald, Germany \\ 2 Yerevan Physics Institute, Alikhanian Brothers Str. 2, 0036 Yerevan, Armenia \\ ${ }^{*}$ Corresponding author: mary@yerphi.am \\ 3 Present address: Forschungszentrum Jülich GmbH, Jülich Centre for Neutron Science (JCNS), Outstation at MLZ, \\ Lichtenbergstrasse 1, 85747 Garching, Germany \\ 4 Pushkov Institute of Terrestrial Magnetism, Ionosphere and Radio Wave Propagation (IZMIRAN), Kalushskoe ave. 4, \\ 142190 Troitsk, Moscow, Russia
}

Received 15 May 2014 / Accepted 24 January 2015

\section{ABSTRACT}

\begin{abstract}
The atmospheric effect correction of the muon flux measured by ground level telescopes is of special importance for further study of cosmic ray variations. The Duperier method is used to correct atmospheric effects on the muon intensity observed by the MuSTAnG telescope. Linear multiple correlation and regression analysis are applied to the data registered during the year 2009. The aerological data are obtained from daily radiosonde balloon flights of Deutscher Wetterdienst. The regression coefficients and total correlation coefficients are calculated for all directional channels. The seasonal variations are eliminated from the MuSTAnG telescope data. The results are compared with theoretical elimination of temperature variations.
\end{abstract}

Key words. Space weather - muon telescope - atmospheric effect

\section{Introduction}

The Muon Space Weather Telescope for Anisotropies (MuSTAnG) (Jansen et al. 2001; Hippler et al. 2008) is presently operating at Greifswald University in Germany to study variations in cosmic rays muon flux. The count rate variation in such instruments is used to study a variety of solar and heliospheric phenomena. However, the wide use of muon detectors for the research of cosmic rays variations is restrained by the presence of atmospheric effects inherent to the muon component of $\mathrm{CR}$.

The investigation of atmospheric effects is of special importance for the further study of cosmic ray variations, since only after the correction for such effects can the measured data provide information on the variations due to causes beyond the Earth's atmosphere.

The two main causes of variations in the cosmic ray flux originating from the Earth's atmosphere are the barometric effect and the temperature effect (Dorman 1974). The barometric effect is determined by only a single parameter, namely the pressure at the detection level. Pressure correction procedures are well established for surface detectors world-wide (Dorman 2004). However, muon observations require additional corrections for the positive and negative temperature effect. Atmospheric temperature effect corrections are correspondingly more complicated. The temperature effect is generally determined by the overall profile of the atmosphere from the level of origin to the detection level, and hence is more difficult to interpret. To exclude the temperature effect, aerologic sounding data near the detector location are necessary. More often such data are missing and it is impossible to restore them in retrospective, or the soundings aren't carried out regularly. Fortunately, there is a weather station in Greifswald (Deutscher Wetterdienst) which routinely takes upper air observations by releasing sounding balloons twice a day at 12:00 and at 24:00 (CEST). These aerological data obtained from the daily radiosonde balloon flights can be used to correct the muon flux measured by the MuSTAnG telescope.

\section{Method}

Usually, the temperature correction procedure is applied after elimination of the pressure effect. There exist different methods (empirical and theoretical) to correct cosmic ray data for atmospheric temperature effects: the method of effective level of generation (Duperier 1949), the integral method (Olbert 1953; Maeda \& Wada 1954; Dorman 1964), the method of effective temperature (Barrett et al. 1952), the method of mass-average temperature (Dvornikov et al. 1976). All these methods depend on the observation of temperature at different altitudes. But we also can get the temperature profile data from global meteorological models, for example the GFS (Global Forecast System, http://www.nco.ncep.noaa.gov/pmb/ products/gfs/) model developed by the National Centers for Environmental Prediction - NCEP (USA). The GFS model's data were used in the temperature effect analysis for the MuSTAnG telescope in previous work (Ganeva et al. 2013). The use of this data allows us to calculate the temperature effect in real time (Berkova et al. 2012).

In this work we will consider corrections according to the Duperier method. It should be noticed that the method used in 

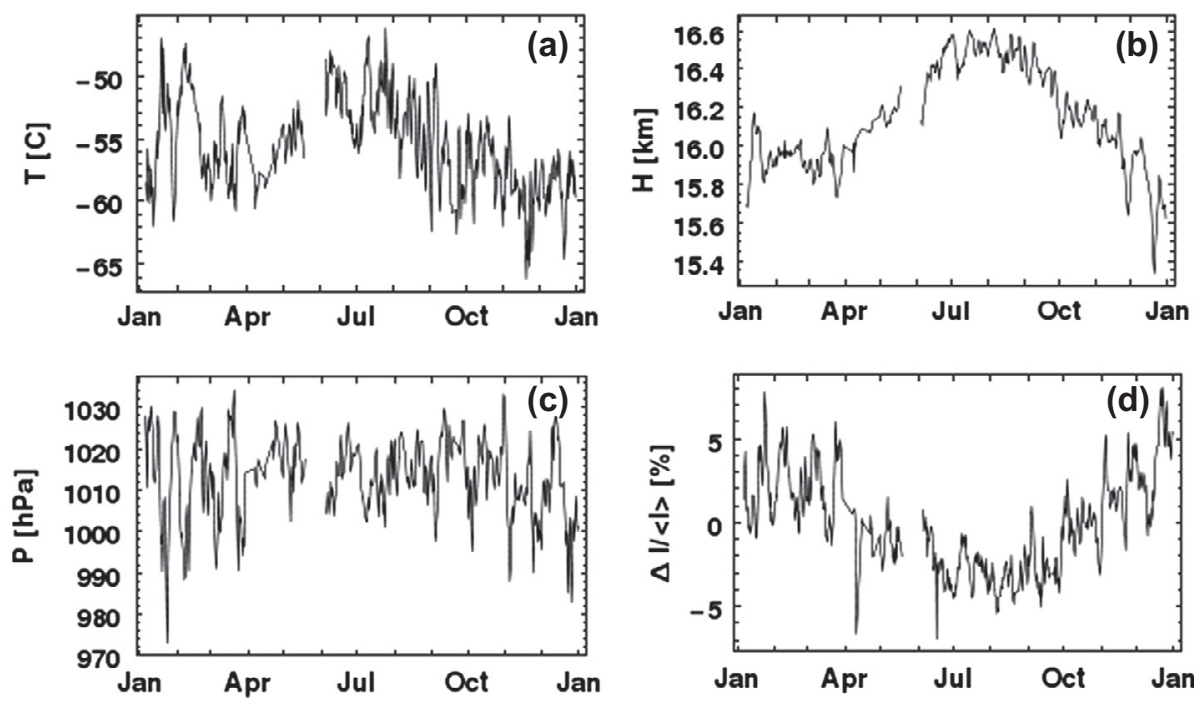

Fig. 1. Variations of the temperature at the $100 \mathrm{hPa}$ level (a); the height of the $100 \mathrm{hPa}$ level (b); the ground pressure (c); muon relative intensity (d) during 2009.

our work allows exclusion of pressure and temperature effect simultaneously, combining pressure, positive temperature and height effects on the muon intensity. Our results will be compared with the results of Ganeva et al. (2013) based on meteorological models.

The Duperier method or the method of effective level of generation is based on the assumption that muons are generated around the isobaric level $100 \mathrm{mb}$. The height of this pressure level in the atmosphere varies, particularly seasonally. The transit time through the atmosphere of muons will be longer when this pressure level is located at a higher altitude and more muons will decay before reaching a detector. The increase in height of this level arises from an expansion of the atmosphere when it is warmer and so this effect is known as the negative temperature effect. When the temperature near the pion production level is higher the air density is lower and the likelihood of the pion interacting before it decays into a muon is reduced resulting in higher count rates. This is known as the positive temperature effect (Duldig 2000). At the energies recorded by the ground level detectors (tens of $\mathrm{GeV}$ ) the negative temperature effect dominates, and at underground registration $(>100 \mathrm{GeV})$ the positive temperature effect prevails.

The method of effective level of generation is the simplest methodology of temperature correction and is still useful for properly correcting the temperature effect on a yearly perspective.

Duperier has presented a linear regression equation for the intensity registered on ground during the quiet sun

$$
I=\text { const. }+\alpha \cdot P+\beta \cdot H+\gamma \cdot T \text {. }
$$

The equation for relative variations is then

$$
\Delta I / I=\alpha \cdot \Delta P+\beta \cdot \Delta H+\gamma \cdot \Delta T .
$$

Here, $\alpha$ is the partial pressure coefficient $(\% / \mathrm{hPa}), \beta$ is the height coefficient $(\% / \mathrm{km})$ and $\gamma$ is the positive temperature coefficient $(\% / \mathrm{C}) . P$ is the ground pressure and $H$ and $T$ are the height and the temperature of the reference layer (the level of maximum muon production). $\Delta P$ is the deviation of the pressure, $\Delta H$ and $\Delta T$ are the deviations of the height and the temperature of the reference layer, and $\Delta I$ is the deviation of the muon count rate from their annual averages, respectively.

Generally the temperature effect of the cosmic ray intensity is characterized by one or two terms in Eq. (2) (Braga et al. 2013; Okazaki et al. 2008; Sagisaka 1986):

$$
\Delta I / I=\beta \cdot \Delta H+\gamma \cdot \Delta T .
$$

The full formula is used to simultaneously eliminate pressure, positive temperature and height effects on the muon intensity (Baker et al. 1993).

Having determined a set of corrections coefficients the intensity corrected for atmospheric effects becomes (as function of time):

$$
\begin{aligned}
I_{\text {corr }}= & I(t) /\left(\left[1+\alpha\left(P(t)-P_{0}\right)+\beta\left(H(t)-H_{0}\right)\right.\right. \\
& \left.\left.+\gamma\left(T(t)-T_{0}\right)\right]\right) .
\end{aligned}
$$

Here, $P_{0}, H_{0}$ and $T_{0}$ are the annual averages of the ground pressure, the height and the temperature of the reference layer, respectively.

\section{Analysis}

We have applied a linear multiple correlation and regression analysis to the data registere by the MuSTAnG telescope during 2009. The year 2009 was chosen due to the minimum of solar activity. The aerological data were obtained from daily radiosonde balloon flights (Deutscher Wetterdienst, Weather station Greifswald). As a reference layer the pressure level of $100 \mathrm{hPa}$ was used.

Figure 1 shows the variations of the air temperature at the $100 \mathrm{hPa}$ level (a), height of the $100 \mathrm{hPa}$ level (b), ground pressure (c) and muon relative intensity detected by the vertical channel of the MuSTAnG telescope (d). One can see a clear anti-correlation between the variations in muon rates and the height of the $100 \mathrm{hPa}$ level (negative temperature effect), which predominates typically at ground-based detectors.

We have computed the correlation matrix which represents the correlation between all pairs of variables. The correlation 
Table 1. Correlation coefficients between all pairs of variables for the vertical direction of the MuSTAnG telescope.

\begin{tabular}{llrrr}
\hline \hline & $\Delta P$ & $\Delta H$ & \multicolumn{1}{c}{$\Delta T$} & \multicolumn{1}{c}{$\Delta I / I$} \\
\hline$\Delta P$ & 1.00 & 0.34 & -0.20 & -0.67 \\
$\Delta H$ & 0.34 & 1.00 & 0.35 & -0.89 \\
$\Delta T$ & -0.20 & 0.35 & 1.00 & -0.15 \\
$\Delta I / I$ & -0.67 & -0.89 & -0.15 & 1.00 \\
\hline
\end{tabular}

matrix for the vertical direction is presented in Table 1. This table clearly demonstrates that there is a strong anti-correlation between the variations in muon rates and the height of the $100 \mathrm{hPa}$ level and no significant correlation with the temperature of the $100 \mathrm{hPa}$ level. As can be seen from Table 1, muon rates also show strong anti-correlation with pressure.

\section{Results and discussion}

The regression coefficients and the total correlation coefficient $R$ calculated for all directional channels are presented in Table 2. One can see that the variation in the pressure coefficients between channels is not significant while the variations in the temperature/height effects seem significant.
Since the muon energy does not vary significantly over channels (Hippler \& Zazyan 2012), changes in $\beta$ and $\gamma$ are not related to the muon energy. Determination of the coefficients strongly depends on the accuracy of the data. The ground level pressure is measured quite accurately, while height and temperature of the $100 \mathrm{hPa}$ level may bear large measurement errors. Apparently, the errors in the measured parameters lead to the observed variations of $\beta$ and $\gamma$.

After applying the atmospheric corrections by using the calculated coefficients, the seasonal variation can be eliminated. The results for the vertical direction are shown in Figure 2. The muon intensity $I$ in counts per hour during 2009 is plotted. Comparing the pressure corrected data with the pressure and temperature corrected data, one can see that only the latter one allows us to eliminate seasonal variations.

In Figure 3 we compare our results for the year 2009 (vertical direction) with that of Ganeva et al. (2013), where the effective temperature method is used.

According to this method correlation between temperature and muon intensity can be described by the effective temperature $T_{\text {eff }}$, in which the contributions of all atmosphere levels are accounted for with the proper weights. The relationship between atmospheric temperature fluctuations and muon intensity variations is $\Delta I / I_{0}=\alpha_{\mathrm{T}} \Delta T_{\text {eff }} / T_{\text {eff. }}$. Details can be found in Barrett et al. (1952). In Ganeva et al. (2013) vertical

Table 2. The regression coefficients $(\alpha, \beta$ and $\gamma)$ and the total correlation coefficient $(R)$ calculated for all directional channels of the MuSTAnG telescope.

\begin{tabular}{lcccc}
\hline \hline Channel & $\alpha(\% / \mathrm{hPa})$ & $\beta(\% / \mathrm{km})$ & $\gamma(\% / \mathrm{C})$ \\
\hline V & $-0.124 \pm 0.003$ & $-8.30 \pm 0.10$ & $0.032 \pm 0.007$ \\
N & $-0.129 \pm 0.004$ & $-7.60 \pm 0.13$ & $0.010 \pm 0.009$ & $0.049 \pm 0.009$ \\
S & $-0.123 \pm 0.004$ & $-8.03 \pm 0.13$ & $0.017 \pm 0.009$ \\
E & $-0.126 \pm 0.004$ & $-7.67 \pm 0.13$ & $0.027 \pm 0.010$ & 0.98 \\
W & $-0.128 \pm 0.004$ & $-7.88 \pm 0.16$ & $0.001 \pm 0.010$ & 0.97 \\
EE & $-0.131 \pm 0.005$ & $-7.00 \pm 0.20$ & $0.002 \pm 0.010$ & $0.918 \pm 0.009$ \\
NE & $-0.129 \pm 0.004$ & $-7.11 \pm 0.15$ & $0.003 \pm 0.010$ & 0.97 \\
NN & $-0.132 \pm 0.004$ & $-6.96 \pm 0.13$ & $0.034 \pm 0.010$ & 0.92 \\
NW & $-0.131 \pm 0.004$ & $-7.40 \pm 0.16$ & $0.028 \pm 0.010$ & 0.95 \\
SE & $-0.129 \pm 0.004$ & $-7.64 \pm 0.15$ & $0.054 \pm 0.010$ & 0.95 \\
SS & $-0.126 \pm 0.005$ & $-7.15 \pm 0.18$ & $0.004 \pm 0.009$ \\
SW & $-0.125 \pm 0.004$ & $-7.97 \pm 0.16$ & 0.94 \\
WW & $-0.128 \pm 0.003$ & $-7.40 \pm 0.13$ & 0.95 \\
\end{tabular}

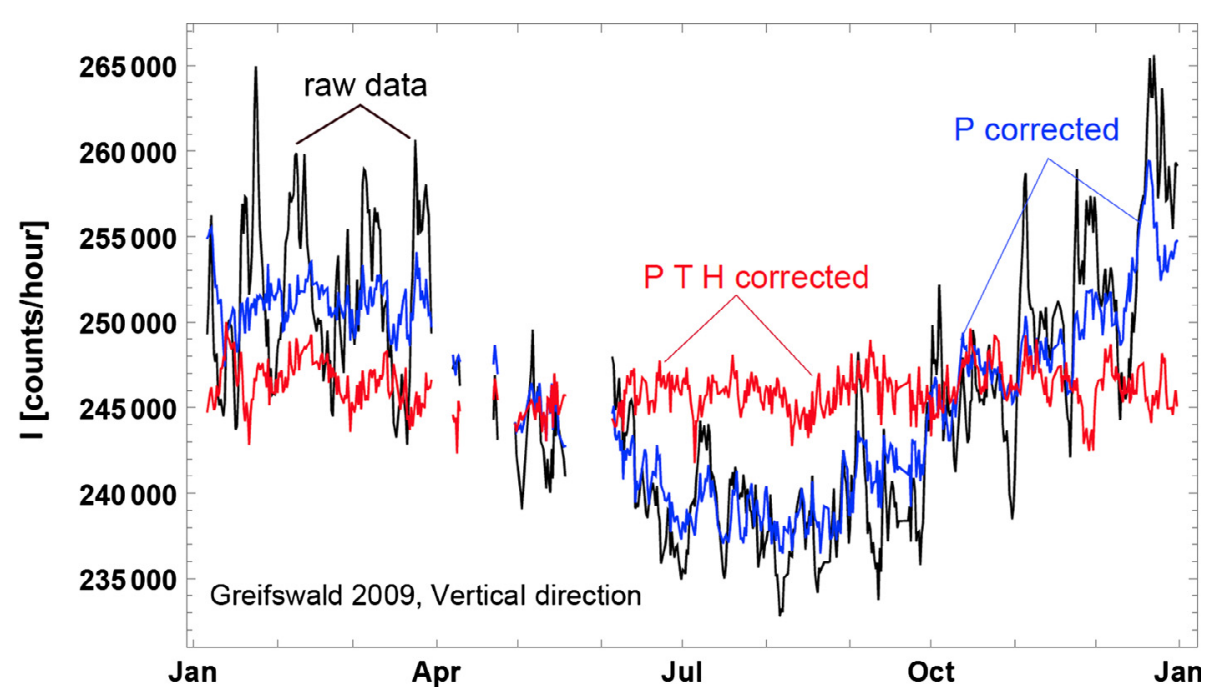

Fig. 2. The muon intensity detected by the vertical channel of the MuSTAnG telescope. 


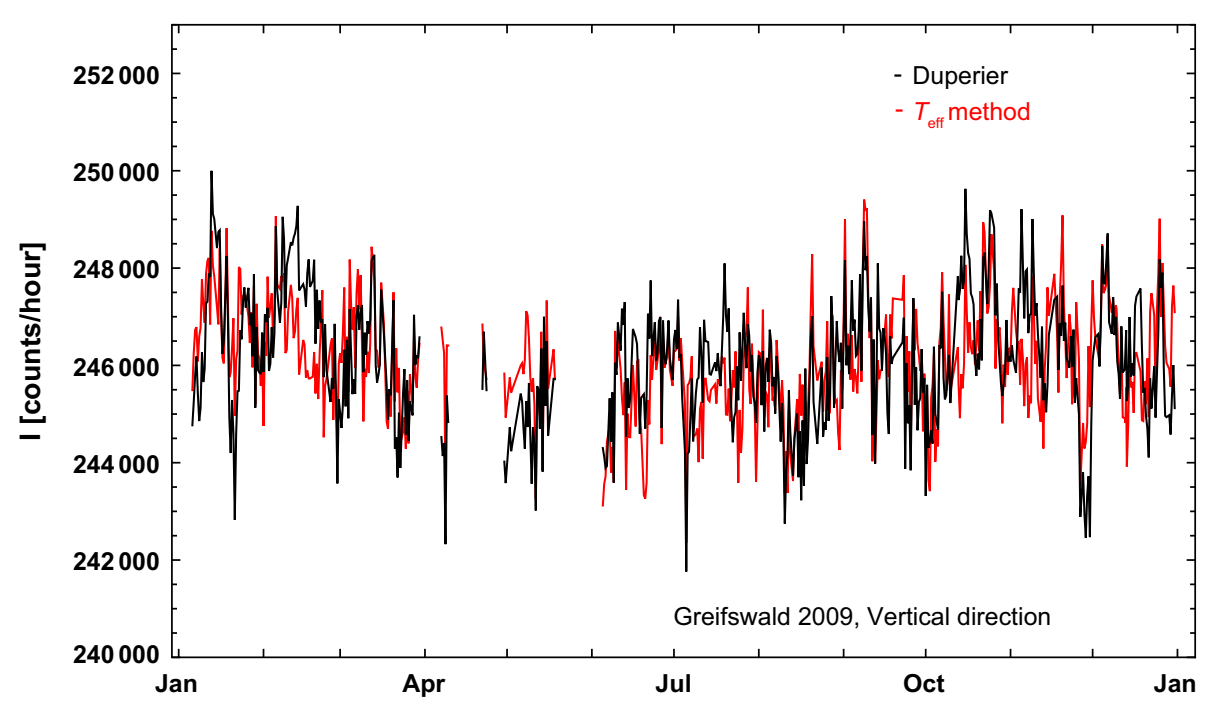

Fig. 3. The muon intensity detected by the vertical channel of the MuSTAnG telescope corrected using the Duperier method with sounding data and the effective temperature method with the GFS model for the temperature calculation.

temperature atmospheric profiles obtained from NCEP's Global Forecast System (GFS) temperature model were used.

To compare results obtained by both of the methods, the experimental temperature measured at the weather station in Greifswald was interpolated by a cubic spline function to obtain hourly data.

Figure 3 shows that both methods result in nearly the same residual fluctuation of the corrected muon rates. Size of the time bin is always one hour. The time of atmospheric measurement is always the start of the muon bin.

\section{Conclusions}

In this work the Duperier method was used to correct for atmospheric effects on the muon intensity observed by the MuSTAnG telescope. The correction coefficients were determined for the base period of the year 2009. The correction of muon intensities was carried out for all directional channels of the MuSTAnG telescope. Corrected muon rates were compared to the results for the elimination of temperature variations obtained by the effective temperature method. It is shown that the Duperier method with three atmospheric variables leads to essentially the same atmospheric corrections of the MuSTAnG telescope intensity as the more complicated effective temperature method applied in Ganeva et al. (2013).

Acknowledgements. Construction of MuSTAnG was supported by the European Space Agency (ESA) and by the Deutsches Zentrum für Luft- und Raumfahrt (DLR).

M. Zazyan thanks The German Academic Exchange Service (DAAD) for providing the opportunity for a research stay at the University of Greifswald.

The editor thanks T. Catanach and an anonymous referee for their assistance in evaluating this paper.

\section{References}

Baker, C.P., D.L. Hall, J.E. Humble, and M.L. Duldig. Atmospheric correction analysis for the Mawson muon telescopes. Proc. of 23th International Cosmic Ray Conference, Calgary, 3, p. 753, 1993.

Barrett, P., et al. Interpretation of cosmic-ray measurements for underground. Rev. Mod. Phys., 24, 133, 1952.

Berkova, M., A. Belov, E. Eroshenko, and V. Yanke. Temperature effect of muon component and practical questions of how to take into account in real time. Astrophys. Space Sci. Trans., 8, 41-44, 2012, http://www.astrophys-space-sci-trans.net/8/41/2012/astra8-41-2012.pdf.

Braga, C.R., A. Dal Lago, T. Kuwabara, N.J. Schuch, and K. Munakata. Temperature effect correction for the cosmic ray muon data observed at the Brazilian Southern Space Observatory in São Martinho da Serra. J. Phys.: Conf. Ser., 409, 012138, 2013.

Dorman, L.I. On the temperature effect of the hard component of cosmic rays. Dokl. Akad. Sci. Nauk SSSR, 95, 49, 1964.

Dorman, L.I. Cosmic rays. Variations and space explorations, North-Holland, Amsterdam, 1974.

Dorman, L.I. Cosmic rays in the Earth's atmosphere and underground, Kluwer Academic Publishers, USA, 2004.

Duldig, M.L. Muon observations. Space Sci. Rev., 93, 207-226, 2000.

Duperier, A. The meson intensity at the surface of the Earth and the temperature at the production level. Proc. Phys. Soc., 62A, 684-696, 1949.

Dvornikov, V.M., Yu.Ya. Krestyannikov, and A.V. Sergeev. Determination of the mass-average temperature on the cosmic ray intensity data. Geomag. Aeron., 16 (5), 923-925, 1976.

Ganeva, M., S. Peglow, R. Hippler, M. Berkova, and V. Yanke. Seasonal variations of the muon flux seen by muon telescope MuSTAnG. J. Phys.: Conf. Ser., 409, 012242, 2013, DOI: $10.1088 / 1742-6596 / 409 / 1 / 012242$.

Hippler, R., A. Mengel, F. Jansen, G. Bartling, W. Göhler, et al. First space weather observations at MuSTAnG - the muon space weather telescope for anisotropies at Greifswald. Proc. of 30th International Cosmic Ray Conference, Mexico, 1, pp. 347-350, 2008.

Hippler, R., and M. Zazyan. Simulation of MuSTAnG telescope response to cosmic rays. Proc. Cosmic Ray Summer 
M. Zazyan et al.: Atmospheric effect corrections of MuSTAnG data

School, Nor-Amberd International Conference Center, p. 30, 2012.

Jansen, F., K. Munakata, M.L. Duldig, and R. Hippler. Muon detectors the real-time, ground based forecast of geomagnetic storms in Europe. ESA Space Weather Workshop: Looking towards a European Space Weather Programme, 2001, ESA WPP-144.

Maeda, K., and M. Wada. Atmospheric temperature effect upon the cosmic ray intensity at sea level. J. Sci. Res. Inst., Tokyo, 48, 71-79, 1954
Olbert, S. Atmospheric effects on cosmic ray intensity near sea level. Phys. Rev., 92, 454, 1953.

Okazaki, Y., A. Fushishita, T. Narumi, C. Kato, S. Yasue, et al. Drift effects and the cosmic ray density gradient in a solar rotation period: First observation with the Global Muon Detector Network (GMDN). Astrophys. J., 681, 693-707, 2008.

Sagisaka, S. Atmospheric effects on cosmic-ray muon intensities at deep underground depths. Nuovo Cimento C, 9, 809, 1986.

Cite this article as: Zazyan, M., M. Ganeva, M. Berkova, V. Yanke \& R. Hippler. Atmospheric effect corrections of MuSTAnG data. J. Space Weather Space Clim., 5, A6, 2015, DOI: 10.1051/swsc/2015007. 